\title{
Perforación duodenal post-CPRE de manejo no quirúrgico: reporte de un caso
}

\section{A Case Report of Non-Surgical Duodenal Perforation Following ERCP}

Gustavo Adolfo Reyes M. ${ }^{1}$, Germán David Carvajal P.2, Robin Germán Prieto 0. ${ }^{3}$, Wilmar Alfredo Martin M. ${ }^{4}$

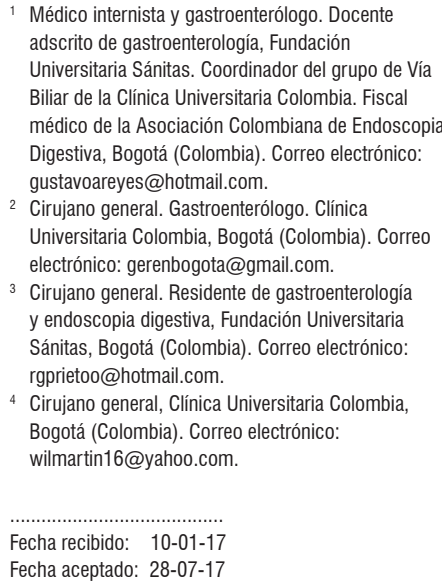

\begin{abstract}
Resumen
La perforación duodenal posterior a la colangiopancreatografía retrógrada endoscópica (CPRE) es una complicación infrecuente que sucede en un $0,1 \%-0,6 \%$ de los casos. El manejo (quirúrgico o no quirúrgico) depende de varios factores. Presentamos el caso de una mujer que sufrió una perforación duodenal post-CPRE manejada conservadoramente con un stent biliar metálico autoexpandible (SMAE) totalmente recubierto y antibióticos, quien no requirió manejo quirúrgico.
\end{abstract}

\section{Palabras claves}

CPRE (colangiopancreatografía retrógrada endoscópica), perforación duodenal, stent biliar.

\section{Abstract}

Post-ERCP duodenal perforations occur in only 0.1 to $0.6 \%$ of ERCP cases. Whether these occurrences are managed with or without surgery depends on several factors. We report the case of a woman who had a postERCP duodenal perforation that was conservatively managed with a fully covered self-expanding metal stent (FCSEMS) and antibiotics who did not require surgical management.

\section{Keywords}

ERCP (endoscopic retrograde cholangiopancreatography), duodenal perforation, biliary stent.

\section{INTRODUCCIÓN}

Las principales complicaciones de la CPRE son: pancreatitis aguda $(3,5 \%)$, sangrado $(1,3 \%)$, perforación $(0,01 \%$ $2,1 \%)$ y colangitis $(<1 \%)(1,2,3)$. La perforación tiene un riesgo de mortalidad de $0,1 \%-1 \%(4,5)$, pero, si hay retraso en el diagnóstico, puede ser hasta del 8\%-23\% $(5,6)$. El tratamiento, dependiendo de varios factores, puede ser médico (nada por vía oral, antibióticos y analgesia endovenosos) o quirúrgico. Presentamos el caso de una paciente con perforación duodenal post-CPRE tipo II manejada con un stent biliar y tratamiento médico, quien presentó una adecuada respuesta.

\section{CASO CLÍNICO}

Mujer de 54 años con diagnóstico de coledocolitiasis recidivante confirmada por colangiografía por resonancia nuclear magnética con cálculo de 14 milímetros en el colédoco proximal (Figura 1). Se realizó CPRE, papilotomía amplia y extracción del cálculo con canastilla de Dormia, un procedimiento complejo debido a la impactación del cálculo (atrapado en la canastilla) en el colédoco intrapapilar. En la radiografía final, se observó aire libre subdiafragmático (Figura 2), por lo que se irrigó medio de contraste en el área papilar, sin observar extravasación. Con el diagnóstico de perforación duodenal, se decidió insertar un stent biliar 
metálico autoexpandible (SMAE) totalmente recubierto de 80 × $10 \mathrm{~mm}$ (Figuras 3 y 4). Se inició manejo con líquidos endovenosos — nada por vía oral-, ampicilina/sulbactam 3 g por vía endovenosa cada 6 horas durante 8 días $\mathrm{y}$ analgesia endovenosa.

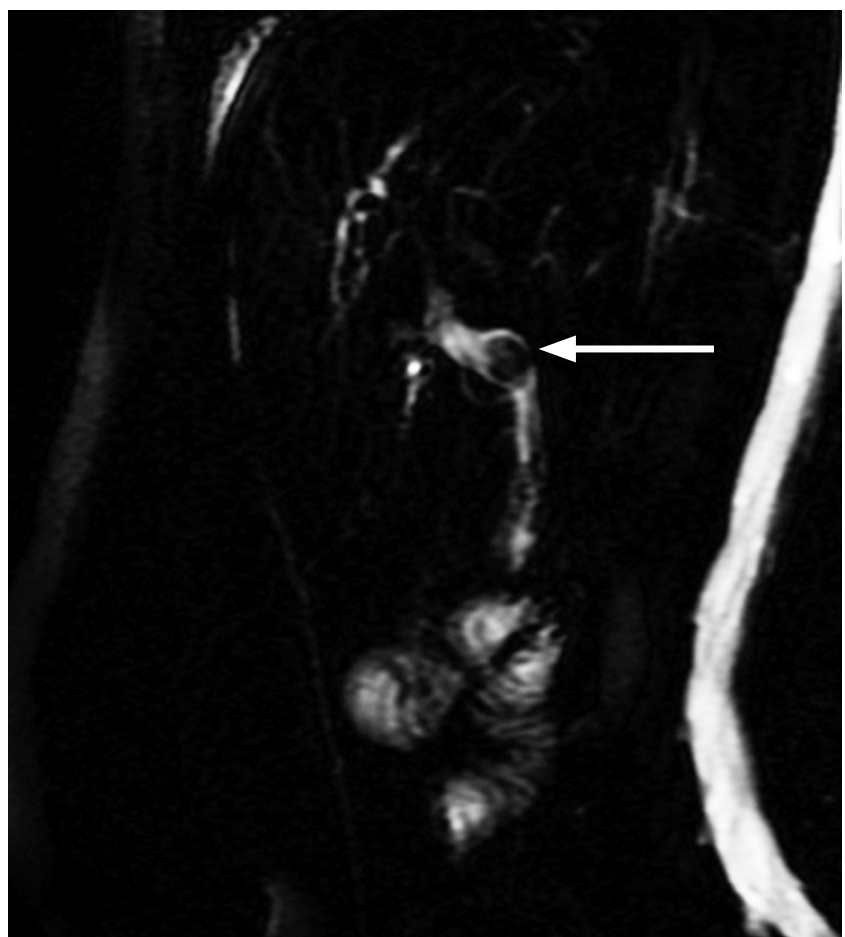

Figura 1. Colangiografía por resonancia nuclear magnética. Cálculo de 14 milímetros en el colédoco proximal (flecha).

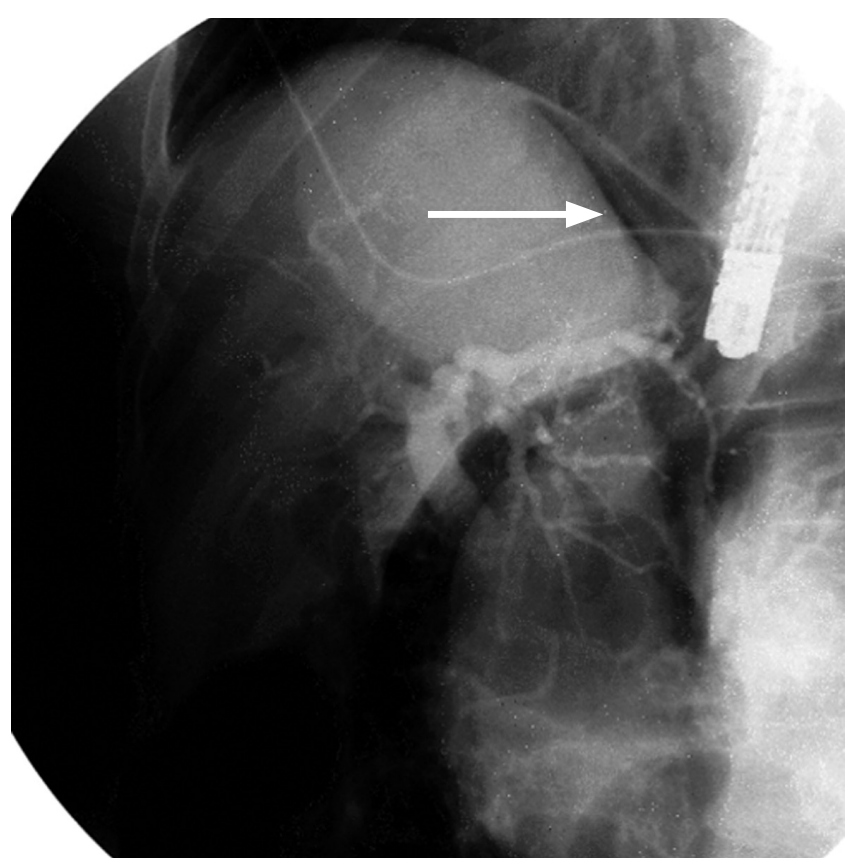

Figura 2. Aire libre subdiafragmático (flecha) luego de la extracción del cálculo.

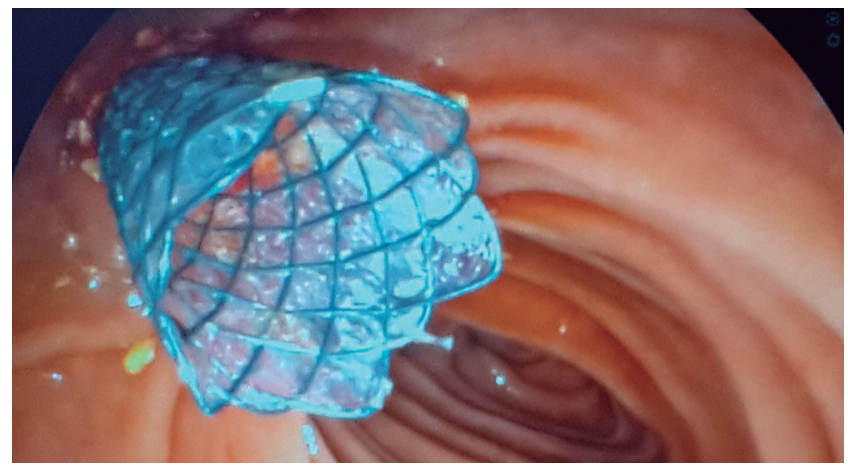

Figura 3. Imagen endoscópica del stent biliar.

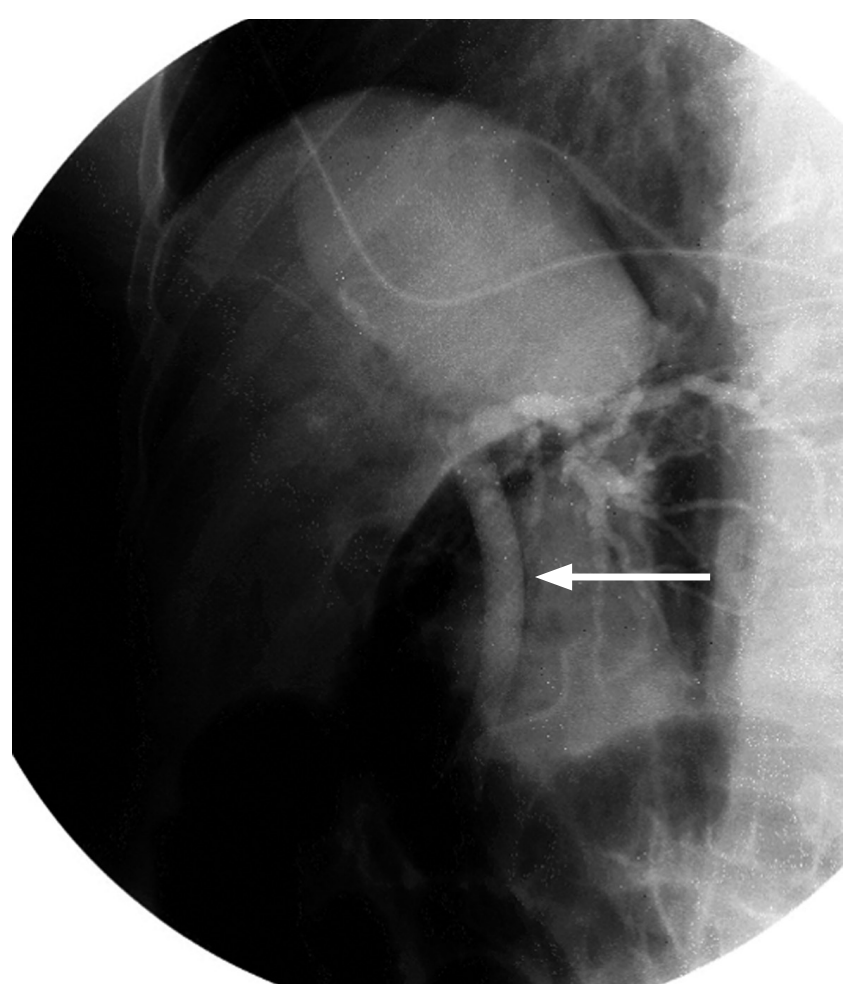

Figura 4. Imagen radiológica del stent biliar (flecha).

Se realizó una tomografía computarizada (TC) de abdomen, que mostró el stent biliar, aire libre perihepático, perirrenal derecho y periduodenal, sin extravasación del medio de contraste (Figuras 5 y 6). La paciente fue valorada por el servicio de cirugía general, y se decidió, en conjunto, seguir el manejo instaurado. En el posoperatorio inmediato, manifestó leve dolor abdominal superior sin signos de irritación peritoneal ni clínica de respuesta inflamatoria sistémica. Durante los cuatro primeros días, presentó leucocitosis y neutrofilia que se normalizaron al quinto día (Tabla 1). Al tercer día, se inició nutrición parenteral total, que fue suspendida al séptimo día dada la tolerancia a la vía oral. Fue dada de alta al octavo día y se retiró el SMAE a las 12 semanas sin complicaciones. 


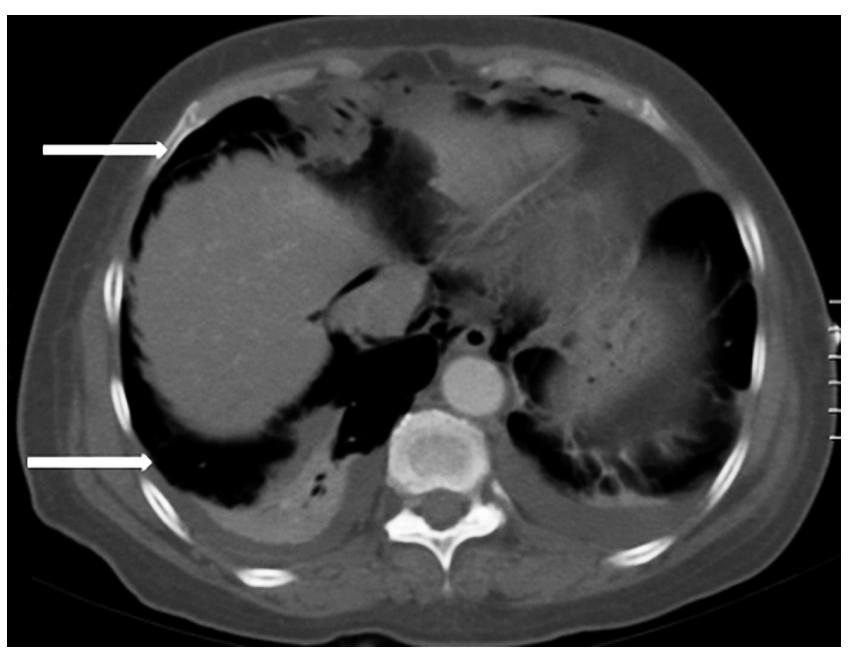

Figura 5. TC en la que se observa aire libre perihepático (flechas).

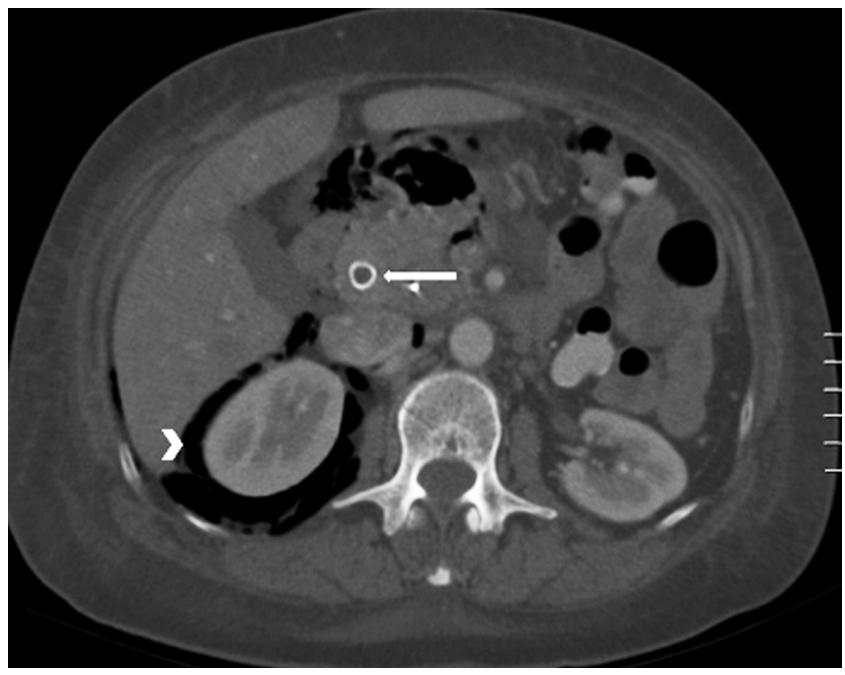

Figura 6. TC en la que se observa aire libre perirrenal derecho (punta de flecha) y el stent biliar metálico (flecha).

Tabla 1. Evolución del recuento de leucocitos.

\begin{tabular}{cl}
\hline Día post-CPRE & Leucocitos/ $\boldsymbol{\mu L}$ y neutrófilos \\
\hline 1 & Leucocitos: 12 270; neutrófilos: $82 \%$ \\
2 & Leucocitos: 14900 ; neutrófilos: $80 \%$ \\
3 & Leucocitos: 15 100; neutrófilos: $81 \%$ \\
4 & Leucocitos: 10500 ; neutrófilos: $79 \%$ \\
5 & Leucocitos: 8820 ; neutrófilos: $65 \%$ \\
\hline
\end{tabular}

\section{DISCUSIÓN}

Howard presentó, en 1999, una de las primeras clasificaciones de perforación post-CPRE (7). Actualmente, la más utilizada es la de Stapfer (8), basada en el mecanismo y localización anatómica, la cual orienta hacia el tratamiento quirúrgico o no quirúrgico:

- Tipo I: perforación duodenal en la pared lateral o medial, causada por el duodenoscopio; generalmente, es grande, ocurre lejos de la ampolla y requiere tratamiento quirúrgico.

- Tipo II: perforación periampular causada por la esfinterotomía; generalmente, es pequeña, la fuga de medio de contraste es mínima o nula, no presenta colecciones y requiere cirugía con menos frecuencia. El diagnóstico se puede confirmar mediante TC o radiografía de vías digestivas altas.

- Tipo III: perforación del conducto biliar causada por la guía o por instrumentación con la canastilla; generalmente, son lesiones pequeñas, que no producen colecciones y pueden ser observadas.

- Tipo IV: debido a la compresión sostenida del aire, se causa una microperforación manifestada por la presencia de aire retroperitoneal. No requiere tratamiento quirúrgico.

Otros mecanismos han sido implicados en la perforación: el avance excesivo de la guía (perforación del hígado), el uso de balón extractor o dilatador y la migración de los stents (9). Se han definido factores de riesgo relacionados con el paciente (antecedente de gastrectomía Billroth II) y con la técnica (experiencia del endoscopista, canulación difícil, precorte y esfinterotomía) $(5,10)$.

El diagnóstico de la perforación se puede realizar durante la CPRE. Si se hace más tardíamente puede tener peor pronóstico. Durante el procedimiento se debe prestar mucha atención a factores que hagan sospechar la perforación. Es importante examinar cuidadosamente la papila y la pared duodenal. La perforación retroperitoneal puede causar enfisema subcutáneo y, en la fluoroscopia, se observa alteración en la sombra renal o áreas radiopacas irregulares. La TC confirma la presencia de neumoperitoneo o de colecciones (11).

Existe consenso en cuanto al tratamiento de las perforaciones del tipo III y IV, especialmente cuando son causadas por la guía. Se considera que tienden a un cierre temprano y no necesitan la colocación de stent. Requieren seguimiento clínico y radiológico (10).

En cuanto a las perforaciones tipo II, algunos autores recomiendan un tratamiento quirúrgico temprano (12), pero varios estudios recientes sugieren un tratamiento conservador, aún en casos de perforaciones retroperitoneales, que consiste en la colocación de un drenaje nasobiliar o de un stent biliar, la suspensión de la vía oral, la administración de antibióticos de amplio espectro, la nutrición parenteral y la estricta observación clínica durante 48 horas con realiza- 
ción de estudios tomográficos para evaluar la aparición de colecciones $(10,11,13)$.

La perforación tipo I se puede manifestar como dolor abdominal y vómito (similar a la pancreatitis post-CPRE). Algunos grupos indican tratamiento quirúrgico con la colocación de un tubo en $\mathrm{T}$ en el colédoco, duodenostomía, diverticulización duodenal o exclusión pilórica $(9,15)$. Otros autores, incluidas las guías de la Sociedad Europea de Endoscopia Gastrointestinal (ESGE, por sus siglas en inglés), recomiendan evaluar el mecanismo, la extensión de la lesión y el tiempo trascurrido al momento del diagnóstico para definir un tratamiento conservador, junto con la aplicación de clips, o indicar manejo quirúrgico $(7,14,16)$. En Colombia, en el estudio realizado por Peñaloza y colaboradores en el hospital San José de Bogotá, en 372 pacientes, se encontró una incidencia de perforación post-CPRE del 1,3\% (17). En la serie de 4 casos de perforación postCPRE de Gómez y colaboradores en el hospital El Tunal de Bogotá, se realizó manejo médico exitoso con hemoclips y ligadura con banda en dos de los pacientes (18).

En nuestro caso, la perforación (tipo II) no fue causada directamente por la papilotomía (las imágenes radiológicas pospapilotomía no muestran aire libre), sino que sucedió durante la extracción difícil del cálculo impactado en el colédoco intrapapilar. Durante la tracción forzada de la canastilla, se produjo un desgarro que "aumentó" la longitud de la papilotomía y provocó la perforación, confirmada por la presencia de aire libre subdiafragmático en la imagen posextracción del cálculo. Debido al diagnóstico en el mismo acto endoscópico y a la ausencia de fuga del medio irrigado sobre la papila, se consideró el manejo con el SMAE totalmente recubierto con el doble fin de evitar la extravasación de bilis hacia el retroperitoneo y de sellar la perforación una vez que el stent se expandiera al ser liberado.

\section{CONCLUSIONES}

El manejo conservador con stents biliares es posible en algunos casos de perforaciones duodenales post-CPRE tipo II teniendo en cuenta varios factores, como el diagnóstico temprano (preferiblemente inmediato), el tamaño de la lesión y la disponibilidad de los elementos necesarios para resolver la complicación. Además, siempre debe realizarse con un seguimiento clínico estrecho en conjunto con el cirujano.

\section{Declaración de conflictos de interés}

Ninguna.

\section{Financiación}

Ninguna.

\section{REFERENCIAS}

1. Anderson M, Fisher L, Jain R, et al. Complications of ERCP. ASGE Guidelines. Gastrointest Endosc. 2012:75:467-73. Doi: https://doi.org/10.1016/j.gie.2011.07.010

2. Cotton PB, Garrow DA, Gallagher J, et al. Risk factors for complications after ERCP: a multivariate analysis of 11,497 procedures over 12 years. Gastrointest Endosc. 2009;70:808. Doi: https://doi.org/10.1016/j.gie.2008.10.039

3. Tavusbay C, Alper E, Gökova M, et al. Management of perforation after endoscopic retrograde cholangiopancreatography. Ulus Travma Acil Cerrahi Derg. 2016;22(5):441-8. Doi: https://doi.org/10.5505/tjtes.2016.42247

4. Christensen M, Matzen P, Schulze S, et al. Complications of ERCP: a prospective study. Gastrointest Endosc. 2004;60(5):721-31. Doi: https://doi.org/10.1016/S00165107(04)02169-8

5. O'Neil N. Management of duodenal perforation post-endoscopic retrograde cholangiopancreatography. When and whom to operate and what factors determine the outcome? A review article. Journal of the Pancreas. 2011;13(1):18-25.

6. Krishna RP, Singh RK, Behari A, et al. Post endoscopic retrograde cholangiopancreaticography perforation managed by surgery or percutaneous drainage. Surg Today. 2011;41:6606. Doi: https://doi.org/10.1007/s00595-009-4331-z

7. Howard TJ, Tan T, Lehman GA, et al. Classification and management of perforations complicating endoscopic sphincterotomy. Surgery. 1999;126:658-65. Doi: https:// doi.org/10.1016/S0039-6060(99)70119-4

8. Stapfer M, Selby RR, Stain SC, et al. Management of duodenal perforation after endoscopic retrograde cholangiopancreatography and sphincterotomy. Ann Surg. 2000;232:191-8. Doi: https://doi.org/10.1097/00000658200008000-00007

9. Kumbhari V, Sinha A, Reddy A, et al. An Algorithm for the management of ERCP-related perforations. Gastrointest Endosc. 2016;83:934-43. Doi: https://doi.org/10.1016/j. gie.2015.09.039

10. Wu H,Dixon E, May G, et al. Management of perforation after endoscopic retrograde cholangiopancreatography (ERCP): a population-based review. HPB. 2006;8:393-9. Doi: https://doi.org/10.1080/13651820600700617

11. Li G, Chen Y, Zhou X, el al. Early Management experience of perforation after ERCP. Clinical Study Gastroenterology Research and Practice. 2012;657418:1-6. Doi: https://doi. org/10.1155/2012/657418

12. Booth FV, Doerr RJ, Khalafi RS, et al. Surgical management of complications of endoscopic sphincterotomy with precut papillotomy. Am J Surg. 1990;159:132-5. Doi: https://doi. org/10.1016/S0002-9610(05)80618-X

13. Ozgonul A, Cece H, Sogut O, et al. Pneumoperitoneum, pneumoretroperitoneum and bilateral pneumothorax caused by ERCP. Journal of the Pakistan Medical Association. 2010;60:60-1.

14. Harvey JP. Pneumopericardium, pneumomediastinum, pneumoretroperitoneum and surgical emphysema secon- 
dary to a duodenal perforation postendoscopic retrograde cholangiopancreatography. BMJ Case Rep. 2015. Doi: https://doi.org/10.1136/bcr-2015-209920

15. Turner R, Steffen C, Boyd P. Endoscopic duodenal perforation: surgical strategies in a regional center. World Journal of Emergency Surgery. 2014;9:11. Doi: https://doi. org/10.1186/1749-7922-9-11

16. Paspatis GA, Dumonceau JM, Barthet M, et al Diagnosis and management of iatrogenic endoscopic perforations:
European Society of Gastrointestinal Endoscopy (ESGE) Positions Statement. Endoscopy. 2014;46(8):693-711. Doi: https://doi.org/10.1055/s-0034-1377531

17. Peñaloza-Ramírez A, Leal-Buitrago $C$, Rodríguez-Hernández A. Adverse events of ERCP at San José Hospital of Bogotá (Colombia). Rev Esp Enferm Dig. 2009; 101:837-49.

18. Gómez MA, Viveros DA. Perforaciones en la CPRE: una complicación para tener en cuenta. Rev Col Gastroenterol. 2014;29:174-8. 


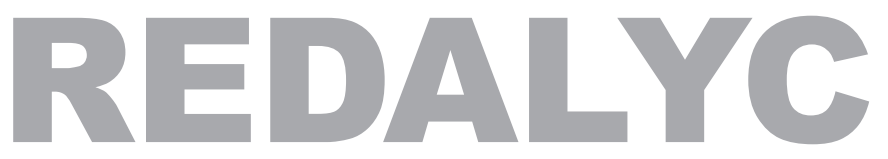

